\title{
Riego Deficitario por Goteo a Niveles de Humedad del Suelo en la Papa (Solanum Tuberosum L.) Variedad Victoria, en el Entorno Geográfico de Riobamba, Ecuador
}

\author{
Robinson Peña, MSc. en Recursos Hídricos \\ Julia Velastegui, MSc. in Geospatial Technologies \\ Escuela Superior Politécnica de Chimborazo, Ecuador
}

Doi: 10.19044/esj.2019.v15n18p238 URL:http://dx.doi.org/10.19044/esj.2019.v15n18p238

\section{Resumen}

En la búsqueda de la eficiencia de uso del agua, describimos la respuesta del cultivo de papa (Solanum tuberosum L.) variedad victoria en Riobamba-Ecuador, con el objetivo de valorar la aplicabilidad de técnicas de "riego deficitario con diferentes niveles de humedad en el suelo", mediante riego localizado, permitiendo dotar láminas de riego en función de tres tratamientos: $25 \%, 50 \%$ y $75 \%$ de agotamiento entre los niveles de capacidad de campo y punto de marchitez permanente. Empleándose la prueba de Scheffé, con diseño experimental de bloques completos al azar con tres repeticiones. Las necesidades hídricas a partir de la curva de coeficiente de cultivo ajustado $\left(\mathrm{K}_{\mathrm{c}}\right)$, mediante el establecimiento de un lisímetro de drenaje por tratamiento y una estación meteorológica, además del registro de la humedad del suelo. El comportamiento de la humedad fue 12,25\%; 10,5\% y $8,75 \%$ para el tratamiento 1,2 y 3 respectivamente. Los Kc para cada etapa fisiológica, fueron para la etapa inicial 0,$35 ; 0,36 ; 0,41$; para la etapa de desarrollo 0,$76 ; 0,80 ; 0,87$; para la etapa intermedia 0,$95 ; 0,98 ; 1,02$; para la etapa final 0,$58 ; 0,59 ; 0,63$ para el tratamiento 1,2 y 3 respectivamente. El máximo nivel de humedad permisible para la variedad es hasta el $50 \%$.

Palabras clave: Riego por goteo, Riego deficitario, Lisímetro, Humedad del suelo 


\title{
Deficit Irrigation by Drip at Soil Moisture Levels in Potato (Solanum Tuberosum L.) Victory Variety, in the Geogrpahical Space Riobamba, Ecuador
}

\author{
Robinson Peña, MSc. en Recursos Hídricos \\ Julia Velastegui, MSc. in Geospatial Technologies
}

Escuela Superior Politécnica de Chimborazo, Ecuador

\begin{abstract}
In the search for the efficiency of water use, we describe the response of the potato crop (Solanum tuberosum L.) victory variety in RiobambaEcuador, with the objective of assessing the applicability of techniques of "deficit irrigation with different levels of moisture in the soil ", by means of localized irrigation, allowing to provide irrigation sheets according to three treatments: $25 \%, 50 \%$ and $75 \%$ depletion between the field capacity levels and permanent wilting point. Using the Scheffé test, with experimental design of randomized complete blocks with three repetitions. The water needs from the adjusted crop coefficient curve $(\mathrm{Kc})$, through the establishment of a drainage lysimeter by treatment and a weather station, in addition to the soil moisture register. The behavior of the humidity was 12,25\%; 10,5\% and $8,75 \%$ for treatment 1,2 and 3 respectively. The Kc for each physiological stage were for the initial stage 0,$35 ; 0,36 ; 0,41$; for the development stage 0,$76 ; 0,80 ; 0,87$; for intermediate stage 0,$95 ; 0,98 ; 1,02$; for the final stage 0,$58 ; 0,59 ; 0,63$ for treatment 1,2 and 3 respectively. The maximum permissible moisture level for the variety is up to $50 \%$.
\end{abstract}

Keywords: Drip irrigation, Deficit irrigation, Lysimeter, Soil moisture

\section{Introduction}

La papa (Solanum tuberosum L.) es uno de los principales cultivos tradicionales, orientado al consumo de la población, ocupando el cuarto lugar a nivel mundial, el mismo que se cultiva en 19 millones de hectáreas, con una producción anual de alrededor de 325 millones de toneladas (FAO, 2016). Mencionar que los países en desarrollo producen más de la mitad de la producción total mundial de papa (FAO, 2009) mismos que han expandido sus áreas de cultivo, sin considerar que en cierta medida esta expansión se vea afectado negativamente por el cambio climático global (Thiele et al., 2010).

En tal sentido se prevé que en el periodo 2010 - 2039 se produzca una reducción de 18 a $32 \%$ de la productividad, trayendo consigo mayor 
variabilidad de las precipitaciones y demanda evaporativa (Hijmans, 2003; Kundzewicz et al., 2007).

Si bien la elevada incidencia de plagas y enfermedades hasta un punto son responsables de los bajos rendimientos, el principal factor limitante del rendimiento y calidad es el estrés hídrico, estimándose que el rendimiento se puede incrementar en aproximadamente un 50\% optimizando el suministro de agua al cultivo (Kumar et al., 2007).

Por consiguiente, los bajos rendimientos de los cultivos ha sido una experiencia común en el país y aun en zonas que depende solo de la precipitación, siendo los agricultores las principales víctimas de la sequía; en tal sentido el desarrollo de técnicas de riego como el riego deficitario, aplicadas en numerosos cultivos de todo el mundo, incluida la papa, con el propósito de alterar la fisiología de la planta y explotar eficientemente las señales hidráulicas y químicas para obtener beneficios agronómicos de interés comercial como pueden ser: controlar un excesivo vigor, incrementar la calidad del fruto, ahorrar agua y mejorar la eficiencia en el uso del agua (Santos et al., 2007; Camposeo \& Rubino, 2003).

\section{Metodología}

\section{Análisis geográfico del área de estudio}

La presente investigación se realizó en los predios del Centro Experimental del Riego (CER) de la Facultad de Recursos Naturales de la Escuela Superior Politécnica de Chimborazo (ESPOCH), Cantón Riobamba, Provincia de Chimborazo, Ecuador, a una altitud de 2821 m.s.n.m. Latitud: 1'39'18,82''S Longitud: 78²0'39,99' W, coordenadas: UTM 17S Datum WGS84, X:758232 Y:9817776. La temperatura media es de $13,5{ }^{\circ} \mathrm{C}$, una

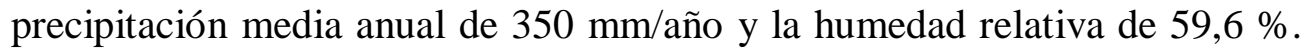
Además según Holdrige (1982), la localidad se encuentra en el rango de estepa espinosa Montano Bajo (eeMB). Los suelos del CER son de una textura franco arenosa, con una capacidad de campo (CC) de 14\%, punto de marchitez permanente (PMP) de 7\%, agua útil $7 \%$ y densidad aparente de $1,5 \mathrm{~g} / \mathrm{cm}^{3}$, en referencia a la calidad del agua posee un $\mathrm{pH} 8,7$ alcalino, una conductividad eléctrica $940 \mu \mathrm{S}$ y una turbidez alta 0,9 .

Como materiales se utilizaron semilla de papa certificada, lisímetros de drenaje, sistema de riego a goteo, tanque evaporación clase A, estación meteorológica portátil, tensiómetros. Con el fin de trabajar con datos alfanuméricos enlazado a datos geoespaciales se utilizó GPS para la toma de coordenadas, cámara fotográfica, software ARGIS, AutoCAD, SPSS, Office, Google Earth, información cartográfica y pluviométrica. 


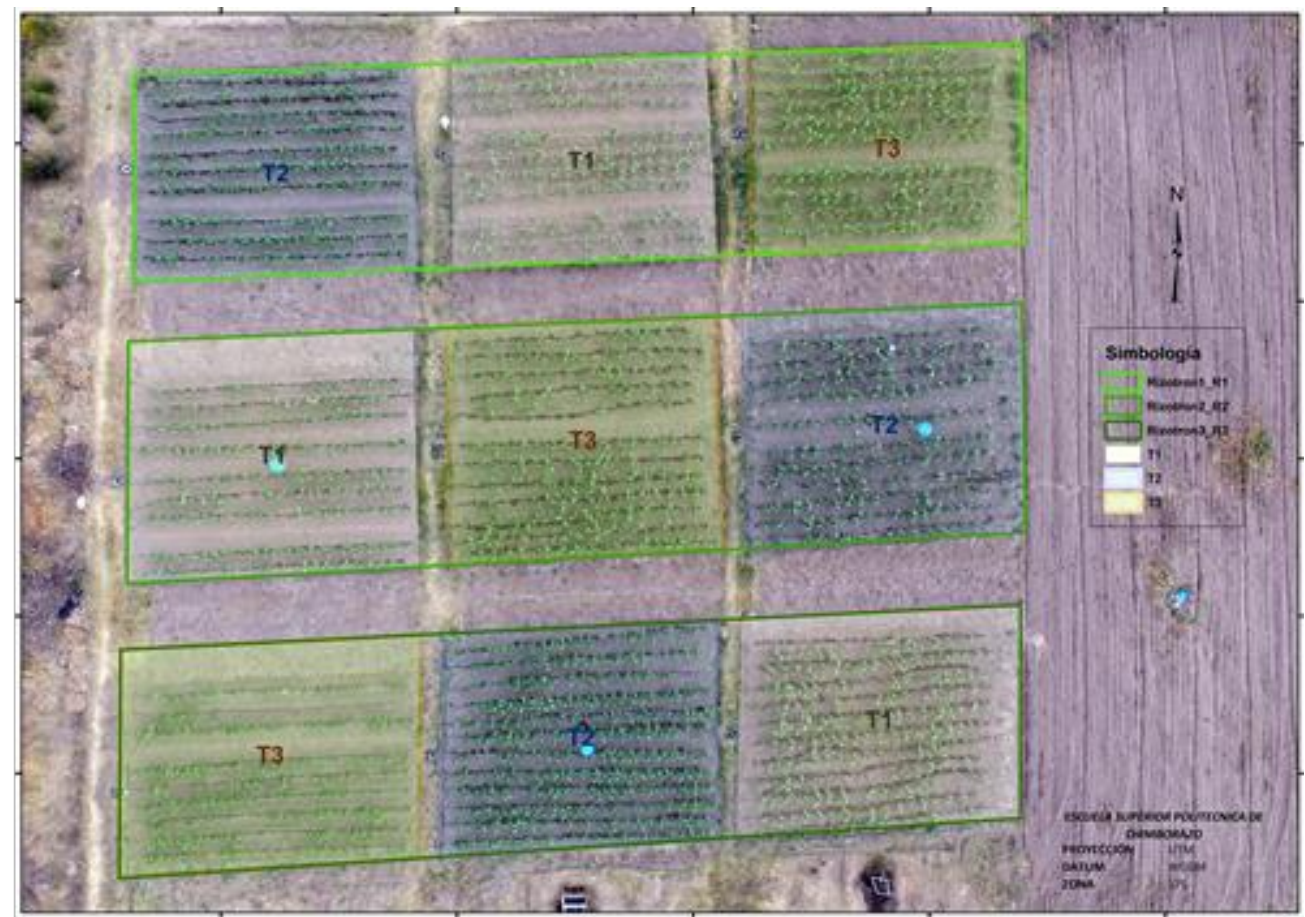

Figura 1.Delimitación del entorno geográfico con sus respectivos elementos de estudio

Como uno de los requerimientos para el desarrollo del presente proyecto fue plasmar la ubicación del área de estudio en mapa a una escala 1:1000, para lo cual se utilizó las coordenadas obtenidas en campo con GPS, ortofoto obtenida en vuelo con UAV. Los insumos antes mencionados fueron utilizados como base para la generación de feature class de los componentes geográficos que se encuentran en el área de estudio, para lo cual se utilizó el software ArcGIS mismo que cuenta con el respectivo licenciamiento educativo. En la Figura 1 se puede visualizar los datos geográficos en formato vectorial tipo polígono (T1, T2, T3, R1, R2, R3) que se generaron utilizando los datos geoespaciales base, lo que permitió delimitar las parcelas del entorno geográfico a ser estudiado. 


\section{Método}

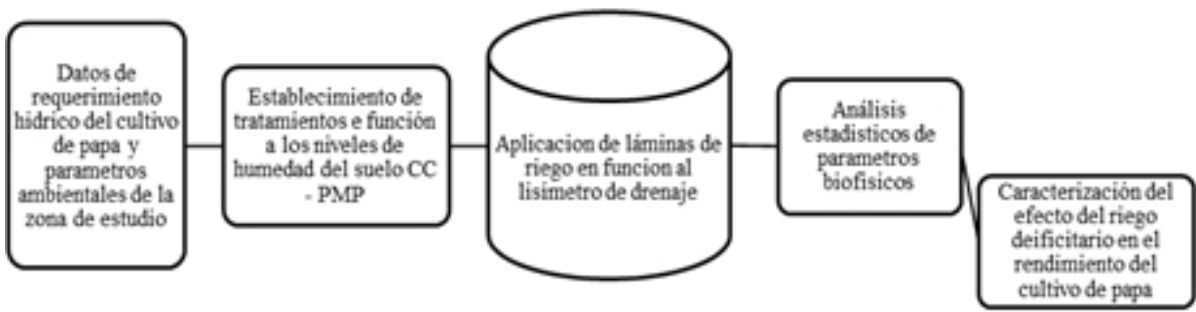

Figura 2. Esquema de los pasos metodológicos para la aplicación de riego deficitario en el cultivo de papa (Solanum tuberosum L.) variedad victoria.

En la Figura 2 se detalla pasos metodológicos que se describen a continuación. La primera fase de la investigación consistió en la recopilación de información base de investigaciones efectuadas por el CER en función al agua de riego y los requerimientos hídricos (Opena \& Porter, 1999), además de lineamientos de la FAO con la determinación de la evapotranspiración del cultivo en condiciones no estándar, es decir bajo condiciones de estrés (Allen et al., 2006). En función de lo mencionado la investigación estuvo compuesta por tres tratamientos $(\mathrm{T} 1=25 \%$ del consumo de agua entre los niveles $\mathrm{CC}$ y PMP, T2 $=50 \%$ del consumo de agua entre los niveles CC y PMP, T3 $=75$ $\%$ del consumo de agua entre los niveles CC y PMP), empleándose un diseño completamente al azar con tres repeticiones con un área por unidad experimental de $100 \mathrm{~m}^{2}$, además dentro de la misma la parcela neta siendo $30 \%$ de la unidad experimental y en si un área total de $1.156 \mathrm{~m}^{2}$. Para los factores e interacciones con significancia estadística se utilizó la prueba de significación de Scheffé al 5\%.

Durante el ciclo de cultivo se llevó a cabo las mediciones biométricas (porcentaje de emergencias, altura de planta, profundidad radicular, tuberización, floración y senescencia) en diez plantas de la parcela neta, en forma semanal. Además se utilizaron sensores para medir temperaturas máximas y mínimas, humedad relativa, precipitación y anemómetro. Además, un tanque estándar evaporímetro tipo A. La cosecha se determinó el número de plantas cosechadas, número de tubérculos por planta, rendimiento por planta, rendimiento total.

\section{Variación de la humedad del suelo}

El porcentaje de humedad se determinó mediante el método gravimétrico, extrayendo una muestra de cada tratamiento a una profundidad de $0-0,30 \mathrm{~m}$, en forma diaria y luego aplicando la ecuación (Enciso et al., 2005; Vasconcelos et al., 2003).

$$
\% \mathrm{H}=\left((\mathrm{Pf}-\mathrm{Ps}) * \mathrm{Ps}^{-1}\right) * 100
$$


Dónde Pf: Peso fresco de la muestra de suelo, Ps: Peso seco de la muestra de suelo.

\section{Medición de la evapotranspiración para los tratamientos}

Tabla 1. Tratamientos en estudio

\begin{tabular}{ll}
\hline Tratamiento & \multicolumn{1}{c}{ Descripción } \\
\hline T1 & $\begin{array}{l}\text { Variedad de papa Victoria con el 25\% del consumo de agua } \\
\text { entre los niveles de CC y PMP. }\end{array}$ \\
T2 & $\begin{array}{l}\text { Variedad de papa Victoria con el } 50 \% \text { del consumo de agua } \\
\text { entre los niveles de CC y PMP. }\end{array}$ \\
T3 & $\begin{array}{l}\text { Variedad de papa Victoria con el } 75 \% \text { del consumo de agua } \\
\text { entre los niveles de CC y PMP. }\end{array}$ \\
\hline
\end{tabular}

Fuente: Peña R. 2019

Para evaluar el efecto del riego deficitario se instaló un sistema de riego por goteo con una manguera auto compensadas con goteros cada $0,3 \mathrm{~m}$ y un caudal de $1.6 \mathrm{~L} / \mathrm{h}$, las mismas que se ubicaron en cada lomo del surco, los riegos se efectuaron cuando se consumió el 25\%, 50\% y 75\% entre los niveles de capacidad de campo (CC) y punto de marchitez permanente (PMP).

Los lisímetros de drenaje, constituye un dispositivo introducido en el suelo con el mismo grado de compactación, el mismo que nos indica la evapotranspiración del cultivo, permitiendo determinar la cantidad de agua a dotar en el siguiente riego es decir la lámina a reponer, aplicando la ecuación (Garay, 2009):

$$
\text { Etc }=\mathrm{R}-\mathrm{D}
$$

Dónde: Etc Evapotranspiración del cultivo, R Agua agregada por lluvia o riegos, D Agua de drenaje durante el periodo de análisis $(D=N 2$ - N1; Siendo $\mathrm{N} 2$ y $\mathrm{N} 1$ niveles de agua en el percolante, al último primer día del periodo considerado).

La evapotranspiración del cultivo de referencia "pasto" (Eto) es una cantidad que depende exclusivamente de las condiciones del medio ambiente misma se determinó aplicando la ecuación (FAO, 2008):

$$
\text { Eto }=\mathrm{Ev} * \mathrm{Kp}
$$

Dónde: Kp, se calcula a partir de datos climáticos de la zona como humedad relativa y viento (Enciso et al., 2005), lo que respecta al volumen a aplicar se calcula aplicando la ecuación (León, 2016):

$$
\mathrm{Va}=(\mathrm{Etc} * \mathrm{ND} * \mathrm{Al}) * \mathrm{c}
$$


Dónde: Va volumen a aplicar, Etc evapotranspiración, ND Número de días que hay de un riego a otro, $\mathrm{Al}$ área del lisímetro, c coeficiente de seguridad para efectuar el riego hasta causar percolación.

El coeficiente de cultivo Kc bajo estrés hídrico ajustado a cada tratamiento en estudio, en función al valor real obtenido con el lisímetro de drenaje se obtuvo a partir de la ecuación (Allen et al., 2006):

\section{Parámetros biofísicos}

$$
\mathrm{Kc}=\mathrm{Etc}^{*} \mathrm{Eto}^{-1}
$$

En función a la guía de toma de datos (Cuesta, 2008), la emergencia se contabilizó en relación al número de plantas emergidas, correlacionándolos con el número de tubérculos sembrados en la parcela neta, expresado en porcentaje.

La altura se midió a partir del cuello hasta el ápice del tallo en diez plantas elegidas al azar de la parcela neta, a los 45, 75 y 105 días después de la siembra (Cuesta, 2008).

La profundidad radicular se midió a partir del cuello de planta hasta la raíz con mayor profundidad, en dos plantas de la parcela neta a los 45, 85 y 110 días después de la siembra (Cuesta, 2008).

\section{Identificación de estados fenológicos}

La senescencia se contó el número de días transcurridos desde la siembra hasta cuando el $50 \%$ de las plantas de la parcela neta presentaron el $50 \%$ de follaje café (Cuesta, 2008). Los valores se expresaron en días después de la siembra (dds).

\section{Rendimiento}

Los tubérculos por planta se tomaron 10 plantas de la parcela neta, de las cuales se contabilizaron el número de tubérculos por planta y se realizó un promedio como dato final (Cuesta, 2008).

La producción por planta se calculó con base al peso total de los tubérculos a la cosecha de la parcela neta, dividida para el número de plantas cosechadas, el promedio se expresó en kg/planta (Cuesta, 2008).

El rendimiento total se determinó cosechando la parcela neta de las tres repeticiones, la misma que se sumó y se sacó un promedio, el resultado se expresará en t/ha (Cuesta, 2008). 


\section{Resultados}

\section{Variación climática durante la fase experimental}

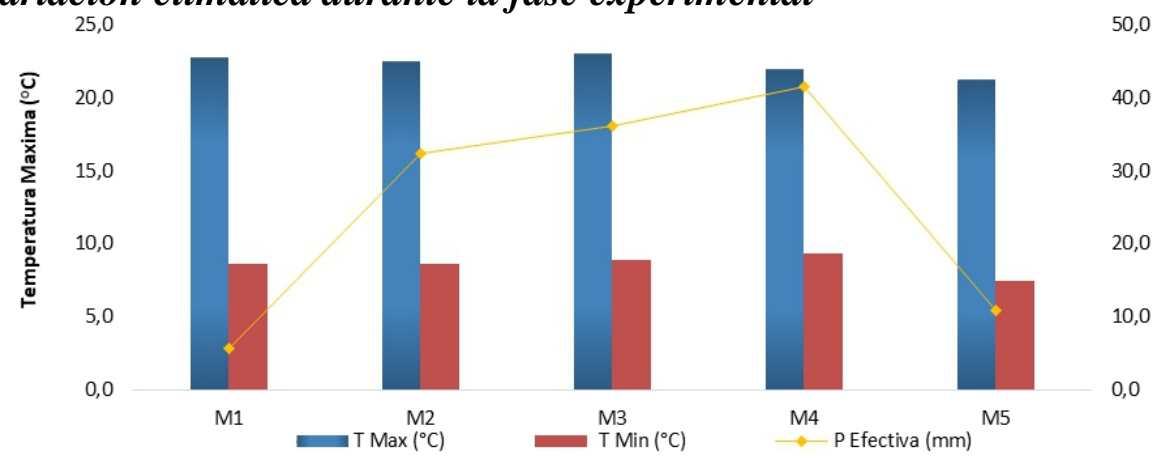

Figura 3. Promedios de temperatura $\left({ }^{\circ} \mathrm{C}\right)$ y precipitación efectiva $(\mathrm{mm})$ durante el ciclo de cultivo de papa (Solanum tuberosum L.) variedad victoria.

En la Figura 3 se observa una fluctuación no relevante de temperatura máxima registrando el mayor valor en el tercer mes con $23,1^{\circ} \mathrm{C}$, y en relación a la temperatura mínima ésta presentó el valor mínimo en el quinto mes con $7,5{ }^{\circ} \mathrm{C}$, cabe mencionar que durante este mes hubo registros de hasta $3{ }^{\circ} \mathrm{C}$, lo que fue necesario tomar las medidas preventivas para evitar daños en el cultivo (FAO, 2008), ya que el cultivo no tolera las temperaturas mínimas bajo los $10{ }^{\circ} \mathrm{C}$ y máximas a $30{ }^{\circ} \mathrm{C}$, mismas que inhabilitan el desarrollo del tubérculo. En referencia a la precipitación efectiva el máximo registrado es 41,4 mm, lo que según (Simpfendofer, 2000), considera que precipitaciones inferiores a $10 \mathrm{~mm}$ no son efectivas para el cultivo ya que estas quedan retenidas en las hojas y se evaporan.

\section{Variación de la humedad del suelo}

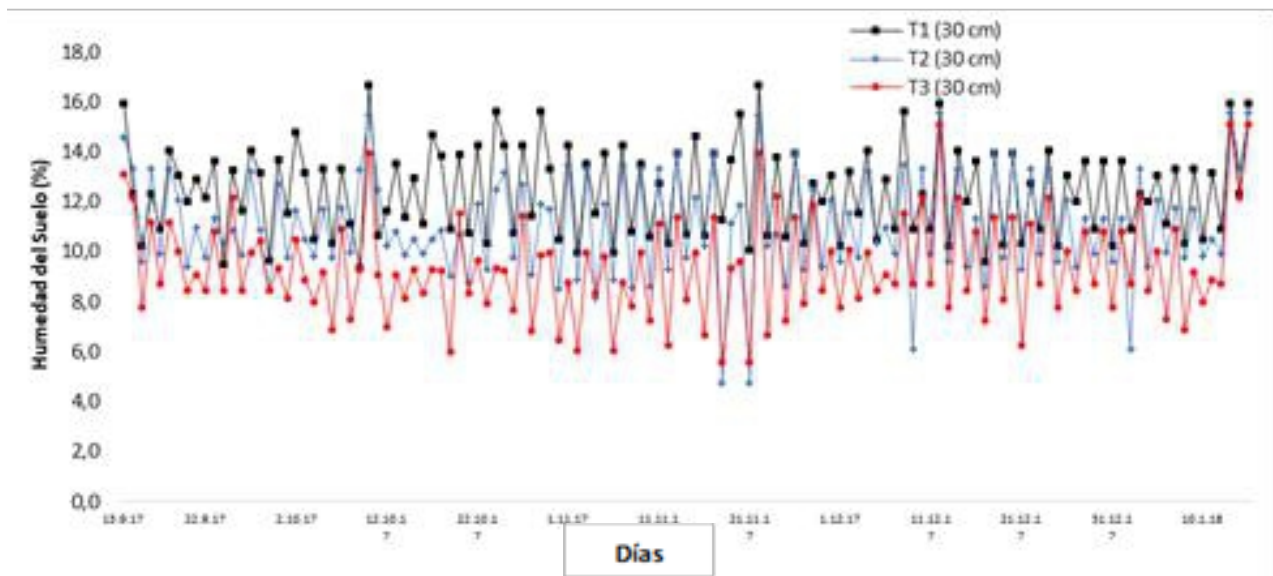

Figura 4. Registro de humedad del suelo a $30 \mathrm{~cm}$ de profundidad, durante el ciclo de cultivo de papa (Solanum tuberosum L.) variedad victoria. 
En la Figura 4. 4 se presenta el comportamiento de la humedad del suelo a la profundidad de $30 \mathrm{~cm}$, donde los patrones de humectación generan la conFiguración del sistema radicular, la planta en cada tratamiento se expuso a diferentes variaciones de humedad del suelo, donde el tratamiento 1 se mantuvo a un nivel de mayor de humedad con un promedio de $12,25 \%$; el tratamiento 2 con $10,5 \%$ y el tratamiento 3 con $8,75 \%$; además estos lapsos de agua ocasionan fisiológicamente un notorio comportamiento de la raíz (Ahmadi et al., 2010; Thompson et al., 2007), que esto influye en la conductividad hidráulica de la raíz a nivel celular, correlacionado con un elevado nivel de ácido abscísico (ABA).

\section{Altura de planta a los 105 días}

Al efectuar el análisis se determinó que a los 45 y 75 días no existía diferencias significativas en altura, caso contrario se evidenció a los 105 días ya que los diferentes niveles de humedad del suelo generó respuestas notorias en la altura de la planta (Figura 5) con un coeficiente de variación de 3,02\%, indicar con otras investigaciones (Deblonde \& Ledent, 2001; Kifle \& Gebretsadikan, 2016), quienes consideran a la altura del tallo como un parámetro trascendental para discriminar la tolerancia a la falta de agua, viéndose reflejado en reprimir la división celular afectando así procesos fisiológicos y bioquímicos (fotosíntesis, traslocación, metabolismo de nutrientes, entre otros) (Farooq, 2008; Jaleel, 2008).

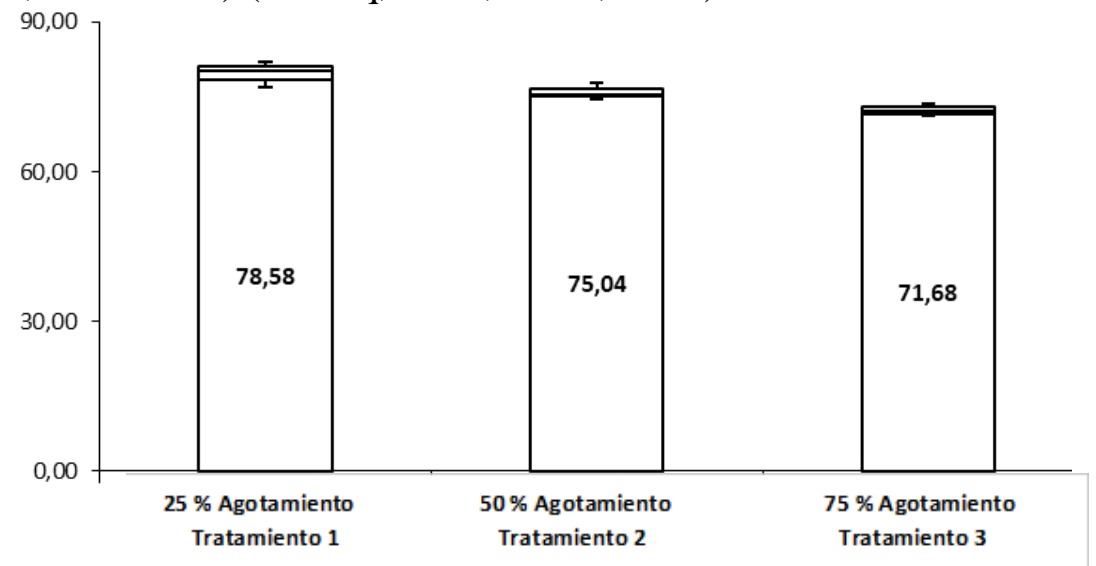

Figura 5. Promedios de altura $(\mathrm{cm})$ a niveles de humedad del suelo en la papa (Solanum tuberosum L.) variedad victoria a los 105 días después de la siembra (dds).

\section{Crecimiento de la raíz a los 110 días}

La evaluación del crecimiento radicular no presentó diferencia significativas a los 45 y 85 días, por el contrario una respuesta notoria se presentó a los 110 días, donde los efectos de la disponibilidad de agua en el suelo en función del tiempo fue significativa, ya que este suelo posee una 
velocidad de infiltración de $21.5 \mathrm{~mm} / \mathrm{h}$, haciendo más visible la respuesta de la planta (Figura 6), presentandose una mayor elongación de la raíz en el tratamiento 3 con $18,5 \mathrm{~cm}$, es así al relacionarlo con otras investigaciones (Stalham \& Allen, 2004), quienes demostraron que a pesar que la densidad de la raíz de la papa disminuye con la profundidad, ya que las raíces de las capas más profundas aportaban notablemente al requerimiento total de agua del cultivo, independiente del estado hídrico de las capas superiores del suelo, además que las raíces jóvenes de la capa profunda presentan una alta eficiencia de extracción de agua (Cuesta, 2008; Kang \& Zhang, 2004). Acotar que dicha eficiencia comparada con otros cultivos no es tan efectiva (Opena \& Porter, 1999). Al presentarse poca disponibilidad de agua en el suelo, la presencia de oxigeno seria mayor que un suelo húmedo, en ese sentido generaría un aumento aparente el crecimiento de la raíz en el suelo seco frente al suelo húmedo (Zhang \& Davies, 1989).

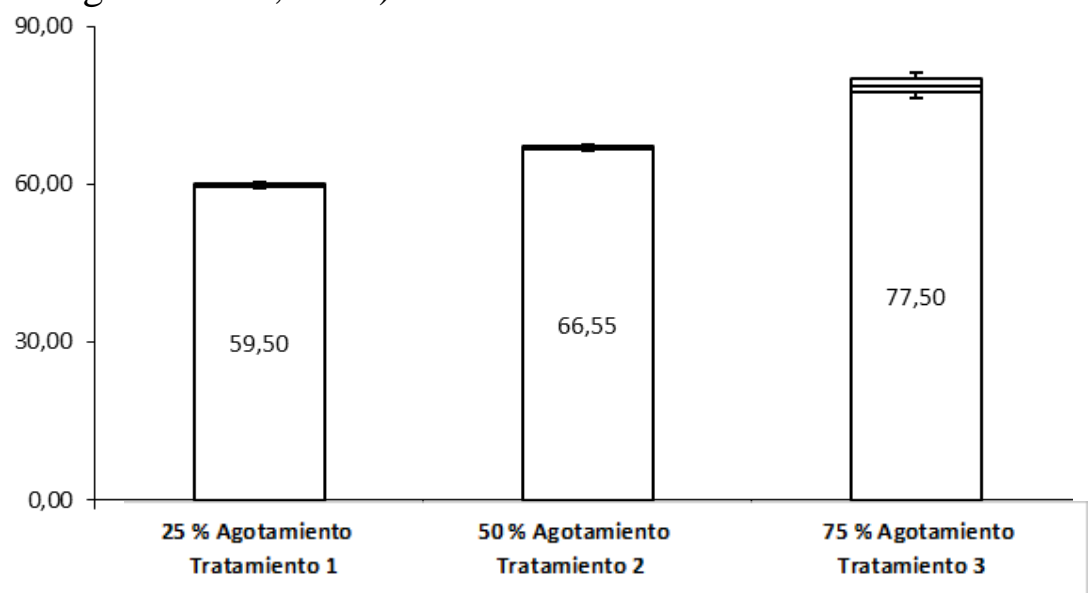

Figura 6. Promedios de raíz $(\mathrm{cm})$ a niveles de humedad del suelo en la papa (Solanum tuberosum L.) variedad victoria a los 110 días después de la siembra (dds)

\section{Días a la senescencia}

En la Figura 7 observamos que el tratamiento 3, es el más precoz con 121,7 días en relación al tratamiento 1 que fue el más tardío con 132 días, es así que el déficit hídrico (Rojas et al., 2007) ocasiona el acortamiento del crecimiento del follaje (madurez temprana del cultivo), con lo cual se reduce el ciclo de vida del cultivo pero si se mantienen condiciones de humedad adecuada alarga el ciclo del cultivo. 


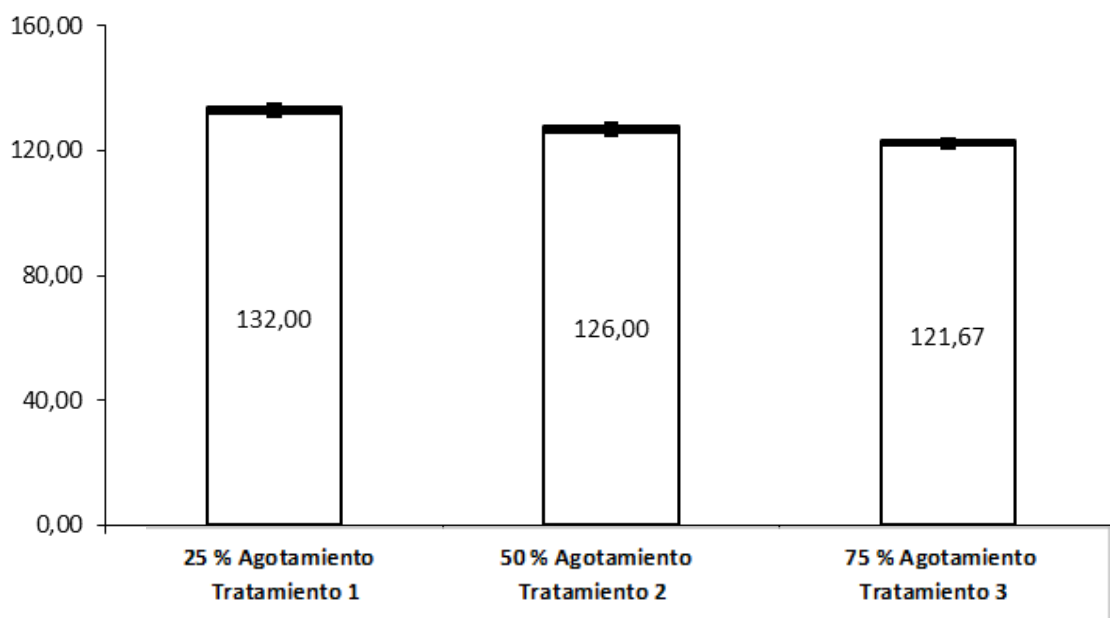

Figura 7. Promedios de días a la senescencia a niveles de humedad del suelo en la papa (Solanum tuberosum L.) variedad victoria.

\section{Coeficiente de cultivo ajustado (Kc) y etapas fenológicas}

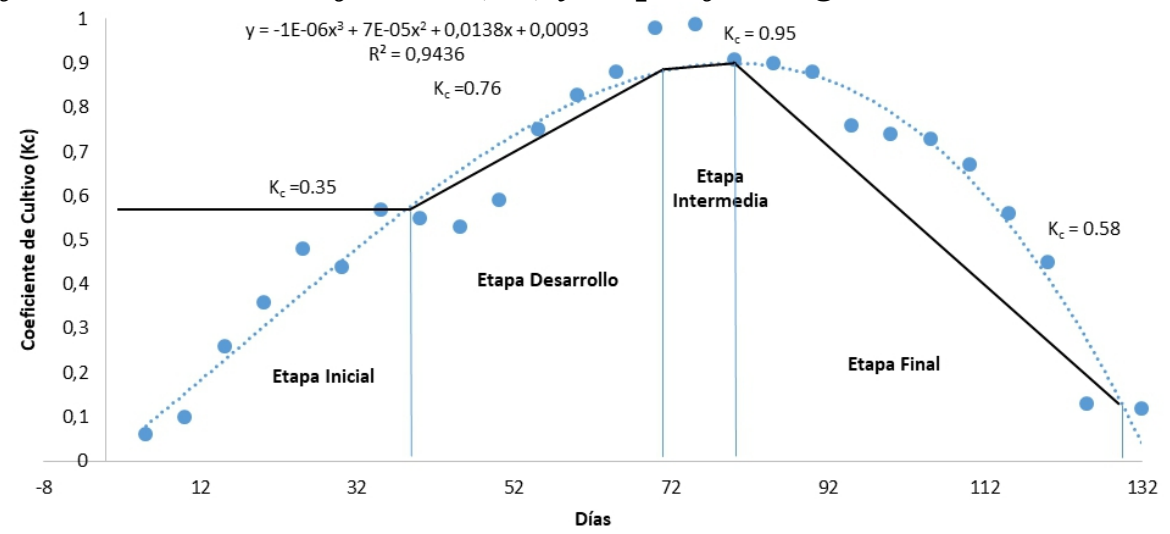

Figura 8. Coeficiente de cultivo ajustado (Kc) del cultivo de papa (Solanum tuberosum L.) variedad victoria para el tratamiento 1 con lisímetro.

El comportamiento del coeficiente de cultivo ajustado $(\mathrm{Kc})$ presenta modelos polinomiales de tercer orden, donde los valores de Kc hallados, varían entre 0,35 y 0,95 para el tratamiento 1 , entre 0,36 y 0,98 para el ,tratamiento 2 y entre 0,41 y 1,02 para el tratamiento 3 . En promedio, los Kc variaron entre 0,38 y 0,98 presentando el valor más alto en la etapa intermedia (Figura 8).

\section{Requerimiento hídrico por etapa fenológica}

El requerimiento hídrico $(\mathrm{mm})$, durante el ciclo de cultivo de papa en cada uno de los tratamientos en estudio se presenta en la Tabla 2. 
Tabla 2. Requerimiento hídrico y lámina de riego en el cultivo de papa (Solanum tuberosum L.) variedad victoria

\begin{tabular}{lllllll}
\hline \multirow{2}{*}{ Etapa } & \multicolumn{2}{l}{ Tratamiento 1 } & \multicolumn{2}{l}{ Tratamiento 2 } & \multicolumn{2}{c}{ Tratamiento 3 } \\
\cline { 2 - 7 } & $\mathbf{R h}$ & $\mathbf{L r}$ & $\mathbf{R h}$ & $\mathbf{L r}$ & $\mathbf{R h}$ & $\mathbf{L r}$ \\
\hline Inicial & 262,42 & 6,31 & 214,12 & 5,96 & 144,85 & 4,87 \\
Desarrollo & 57,72 & 2,88 & 43,46 & 2,73 & 80,4 & 2,98 \\
Intermedia & 15,96 & 3,03 & 12,96 & 2,14 & 9,37 & 2,57 \\
Final & 152,46 & 4,07 & 184,74 & 4,26 & 193,81 & 4,31 \\
Total & 488,56 & 16,29 & 455,28 & 15,09 & 428,43 & 14,73 \\
\hline
\end{tabular}

Rh: requerimiento hídrico (mm); Lr: lámina de riego (mm).

Fuente: Peña R. 2019

Durante el ciclo de cultivo, cada tratamiento presentó efectos significativos en función al grado de crecimiento vegetativo, profundidad radicular y fundamentalmente el rendimiento, llegando la planta hasta un máximo y luego disminuyendo, influyendo las condiciones medioambientales, características del suelo, las prácticas agrícolas y considerablemente el riego aplicado (Beltri, 2008; FAO, 2008).

El requerimiento hídrico es de 488,56 $\mathrm{mm}$ para el tratamiento 1; $455,28 \mathrm{~mm}$ para el tratamiento 2 y $428.43 \mathrm{~mm}$ para el tratamiento 3 , resultados que al comprar con investigaciones (Peña, 2014), indica valores de 409 y 416,7 mm en la zona, considerar que la campaña en que se implementó el ensayo fue condicionante en la necesidad hídrica. Otros investigadores (Sood \& Singh, 2003), mencionan que el requerimiento de agua varía entre 350 y $600 \mathrm{~mm}$, dependiendo del período de duración del cultivo, condiciones ambientales, tipo de suelo y variedad, además la producción disminuye si no se aporta con más del 50 por ciento del total de agua disponible en el suelo durante el crecimiento.

\section{Tubérculos por planta}

La senescencia se contó el número de días transcurridos desde la siembra hasta cuando el 50\% de las plantas de la parcela neta presentaron el $50 \%$ de follaje café. Los valores se expresaron en días después de la siembra (dds). 


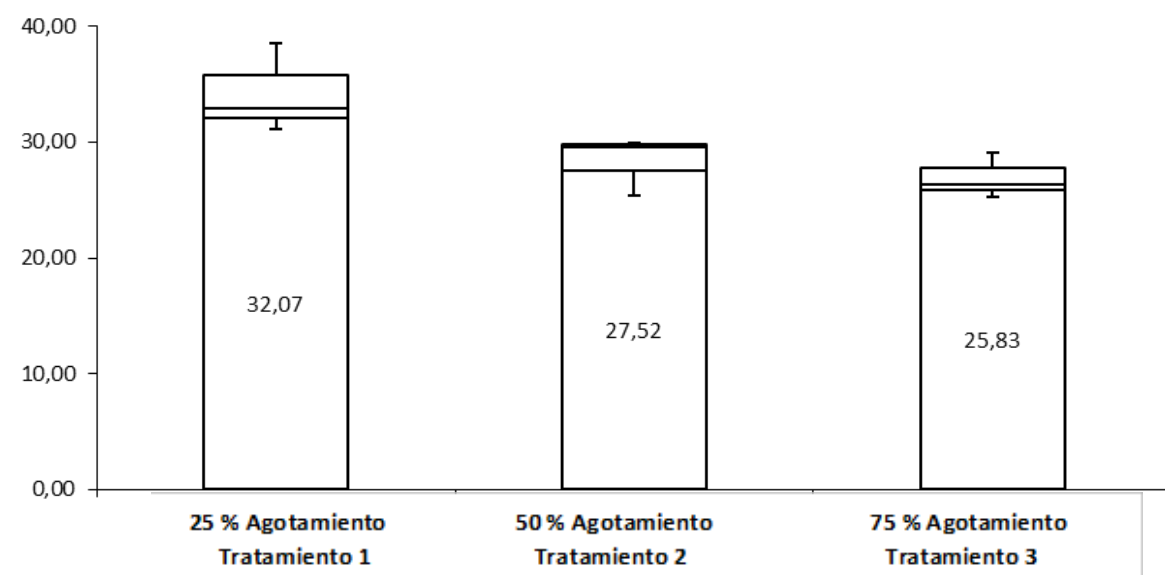

Figura 9. Promedios de tubérculos por planta (u) a niveles de humedad del suelo en la papa (Solanum tuberosum L.) variedad victoria.

Al observar la Figura 9 se observó una diferencia no significativa entre el tratamiento 1 y tratamiento 3, importante considerar al respecto (Kumar et al., 2007; Lahlou \& Ledent, 2005) que al someter a déficit hídrico a la papa, siempre hay tendencia a mayor producción de tubérculos en "cantidad", todo depende también del medio donde se trabaje ya que según trabajos en invernadero reduce el número final de tubérculos por planta.

\section{Producción por planta}

En la Figura 10 según la prueba de Scheffé califica al tratamiento 1 en el rango "a" y al tratamiento 3 en el rango "b" superándolo en casi el doble, considerar que (Simpfendofer, 2000) el impacto económico a la no dotación de agua en los niveles deseados, se ve reflejado en la calidad del tubérculo, además esto dependerá hasta qué punto la planta puede tolerar.

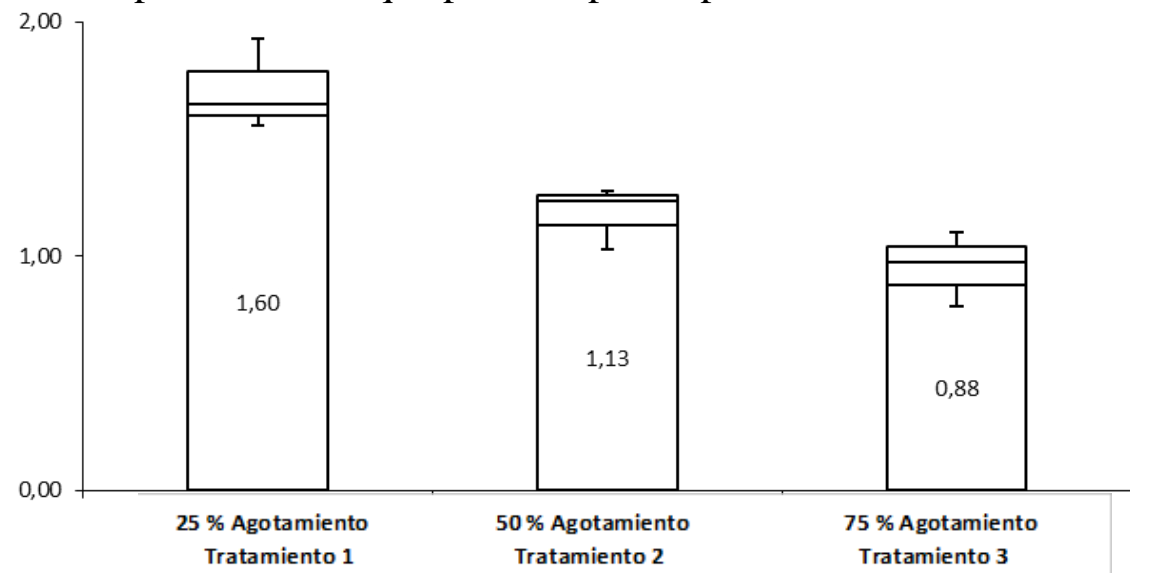

Figura 10. Promedios de rendimiento por planta (kg/planta) a niveles de humedad del suelo en la papa (Solanum tuberosum L.) variedad victoria 
No hubo significancia estadística para los diferentes niveles de humedad esto debido a que las categorías de papas representaban un solo peso (Figura 11), por su parte (Kang \& Zhang, 2004), señala que el contenido de humedad del suelo no debe presentar un umbral menor al $50 \%$ es decir mantener el suelo a capacidad de campo, donde el agua esté disponible para el cultivo sobre todo en etapas críticas, por el contrario se ve afectado el rendimiento del tubérculo directamente al restringir la transpiración y la fotosíntesis e indirectamente al reducir la evaporación del suelo y las hojas, aumentando la temperatura del suelo y de la planta (Zhang \& Davies, 1989).

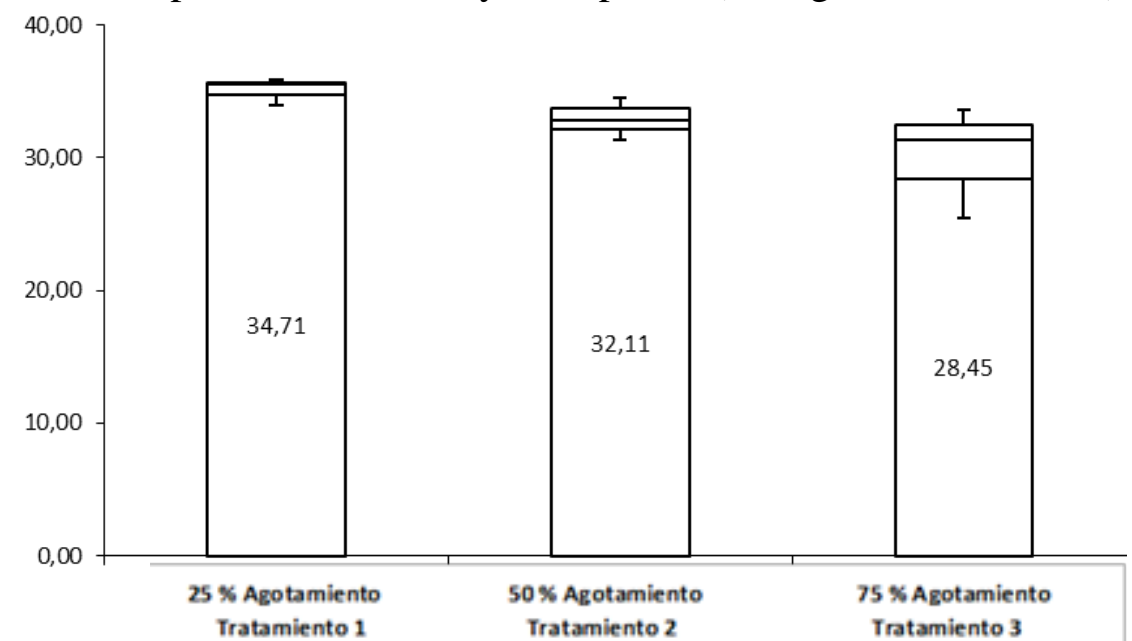

Figura 11. Promedios de rendimiento total (t/ha) a niveles de humedad del suelo en la papa (Solanum tuberosum L.) variedad victoria.

\section{Conclusion}

La altura alcanzada durante el ciclo del cultivo fue de $78,58 \mathrm{~cm}$ para el tratamiento 1 (25\% agotamiento), 75,04 para el tratamiento 2 (50\% agotamiento) y $71,68 \mathrm{~cm}$ para el tratamiento 3 (75\% agotamiento).

Se determinó que el mayor desarrollo radicular alcanzó el tratamiento 3 ( $75 \%$ agotamiento) con una profundidad de $0,78 \mathrm{~m}$; seguido del tratamiento 2 (50\% agotamiento) con $0,67 \mathrm{~m}$ y el tratamiento 1 (25\% agotamiento) con $0,60 \mathrm{~m}$, además destacar que en promedio la mayor concentración de raíces se localizó a los $0,35 \mathrm{~m}$ con un $63 \%$.

El coeficiente de cultivo $(\mathrm{Kc})$ ajustado bajo "estrés hídrico" para la etapa inicial, desarrollo, intermedia y final para el tratamiento 1 (25\% agotamiento) fue 0,$35 ; 0,76 ; 0,95 ; 0,58 ;$ para el tratamiento $2(50 \%$ agotamiento) fue 0,$36 ; 0,80,0,98 ; 0,59$ para el tratamiento $3(75 \%$ agotamiento) $0,41,0,87 ; 1,02 ; 0,63$.

La disponibilidad de agua es directamente proporcional a la duración del ciclo de cultivo destacando para el tratamiento 1 (25\% agotamiento) una duración de 132 días, para el tratamiento 2 (50\% agotamiento) de 126 días y 
para el tratamiento 3 (75\% agotamiento) de 121 días, lo que se concluye que la falta de agua ocasiona que la planta acelere su ciclo fenológico, como una medida de tolerancia ante el estrés hídrico.

El rendimiento total fue de 34,71 t/ha para el tratamiento $1(25 \%$ agotamiento), de 32,11 t/ha para el tratamiento 2 (50\% agotamiento) y 28,45 t/ha para el tratamiento 3 (75\% agotamiento).

\section{References:}

1. Ahmadi, S. H., Andersen, M. N., Plauborg, F., Poulsen, R. T., Jensen, C. R., Sepaskhah, A. R., \& Hansen, S. (2010). Effects of irrigation strategies and soils on field grown potatoes: Yield and water productivity. Agricultural Water Management, 97(11), 1923-1930.

2. Allen, R. G. (2006). Evapotranspiración del cultivo: guías para la determinación de los requerimientos de agua de los cultivos(Vol. 56). Food \& Agriculture Org.

3. Beltri, E. (2008). Medida del balance hídrico y estimación del coeficiente Kc para la mejora de la programación de riegos utilizando técnicas de riego deficitario controlado. Vol 1. 1a ed. Barcelona.

4. Camposeo, S., \& Rubino, P. (2003). Effect of irrigation frequency on root water uptake in sugar beet. Plant and soil, 253(2), 301-309.

5. Cuesta, X. (2008). Guía para el manejo y toma de datos de ensayos de mejoramiento de papa. Estación Experimental Santa Catalina. Instituto Nacional Autónomo de Investigaciones Agropeción y Producción de Raíces y Tubérculos-Papa. Cutuglahua-Pichmcu.

6. Deblonde, P., \& Ledent, J. (2001). Effects of moderate drought conditions on green leaf number, stem height, leaf length and tuber yield of potato cultivars. European Journal of Agronomy, 14(1), 3141.

7. Enciso, J., Porter, D., \& Péries, X. (2005). Sensores de humedad del riego para eficientizar el riego. Cooperativa de Texas, 2-6

8. FAO. (2008). El cambio climático y la producción de alimentos. Vol 10. 6a ed. Roma: Estudio FAO Alimento y Agua. Roma.

9. FAO. (2009). New light on a hidden treasure. Estudio FAO Riego y Drenaje.

10. FAO. (2016). Statistical water. Base de datos FAOSTAT.

11. Farooq, M., Asray, J., \& Mandeal, A. (2008). Función fisiológica de forma exógena aplicada glycinebetaine en la mejora de tolerancia a la sequía de la aromática de grano fino arroz (Oryza sativa L.). J. Agron. Crop Sci, 194, 325-333.

12. Garay, O. (2009). Manual de uso consuntivo del agua para los principales cultivos de los Andes Centrales Peruanos (No. F06 G37F). Instituto Geofísico del Perú, Lima (Peru). 
13. Hijmans, R. (2003). The effect of climate change on global potato production. American journal of potato research, 80(4), 271-279.

14. Jaleel, C. (2008). Estrés hídrico déficit efectos sobre el metabolismo de oxígeno reactivo en Catharanthus roseus. B: Biointerfaces. 62:105111.

15. Kang, S., \& Zhang, J. (2004). Controlled alternate partial root-zone irrigation: its physiological consequences and impact on water use efficiency. Journal of experimental botany, 55(407), 2437-2446.

16. Kifle, M., \& Gebretsadikan, T. (2016). Yield and water use efficiency of furrow irrigated potato under regulated deficit irrigation, AtsibiWemberta, North Ethiopia. Agricultural water management, 170, 133139.

17. Kumar, S., Asrey, R., \& Mandal, G. (2007). Effect of differential irrigation regimes on potato (Solanum tuberosum) yield and postharvest attributes. Indian journal of agricultural science, 77(6), 366368.

18. Kundzewicz Z, Mata L, Arnell N, Döll P, Kabat P, Jiménez B. (2007). Climate change impacts, adaptation and vulnerability. University of Cambridge. 2: 173-210.

19. Lahlou, O., \& Ledent, J. F. (2005). Root mass and depth, stolons and roots formed on stolons in four cultivars of potato under water stress. European Journal of Agronomy, 22(2), 159-173.

20. León-Ruiz, J. (2016). Modelación matemática para estimar los requerimientos hídricos del cultivo de papa (Solanum tuberosum L..). UNALM.

21. Opena, G., \& Porter, G. (1999). Soil management and supplemental irrigation effects on potato: root growth. J. Agron. 91:426-431.

22. Peña, R. (2014). Evaluación de seis genotipos de papa con tolerancia al déficit hídrico Chimborazo Ecuador. En: Ortega S, editores. Papa un alimento milenario. VI Congreso Ecuatoriano de la Papa. pp. 190194.

23. Rojas, G., Posadas, A., Quiroz, R., Holle, M., \& Málaga, M. (2007). Secado parcial de raíces: una promisoria técnica de riego en papa (Solanum tuberosum). J. Water.5: 45 - 56.

24. Santos, T., Lopes, C., Rodrigues, M., De Souza, C., Da-Silva, J., \& Maroco, J. (2007). Effects of deficit irrigation strategies on cluster microclimate for improving fruit composition of moscatel field-grown grapevines. Sci. hortic. 112: 321-330.

25. Simpfendofer. (2000). Efecto del riego en el cultivo de papa: riego y drenaje. INIA. 34:35-38.

26. Sood, M., \& Singh, N. (2003). The potato: production and utilization in sub-tropics. Mehta publishers. J. Water management.32: $121-128$. 
27. Stalham, M., \& Allen, E. (2004). Water uptake in the potato (Solanum tuberosum) crop. Agricultural. 142:373-393.

28. Thiele, G., Theisen, K., Bonierbale, M., \& Walker, T. (2010). Targeting the poor and hungry with potato science. J. Potato. 37: 7586.

29. Thompson, A., Andrews, J., Mulholland, B., Mckee, J., Hilton, H., \& Horridge, J. (2007). Overproduction of abscisic acid in tomato increases transpiration efficiency and root hydraulic conductivity and influences leaf expansion. P. Physiology. 43: 105-117.

30. Vasconcelos, A., Casagrande, D., Perecin, L., \& Landell, J. (2003). Evaluación del sistema radicular de la caña de azúcar por diferentes métodos. J. Root. 12: 56 - 75.

31. Zhang, J., \& Davies, W. (1989). Abscisic acid produced in dehydrating roots may enable the plant to measure water status of the soil. Plant, cell and environment. J. Biotechnol. 5:73-81. 